\title{
First Report of an Outbreak of Contagious Ecthyma in Camels (Camelus dromedarius and Camelus bactrianus) in Iran
}

\author{
Seyed Mohammad Barani ${ }^{1}$, Mohammad Reza Mohebbi ${ }^{2}$, Hamid Reza Varshovi ${ }^{3}$, Amir Niasari-Naslaji ${ }^{4}$, \\ Mohammad Agha-Ebrahimian ${ }^{3}$ and Mohammad Hassan Ebrahimi-Jam³ \\ 1. Iran Veterinary Organization, Qom 3719713846, Iran \\ 2. Department of Internal Medicine, Faculty of Veterinary Medicine, University of Tehran, Tehran 1419963111, Iran \\ 3. Razi Vaccine and Serum Research Institute, Karaj 31975/148, Iran \\ 4. Department of Clinical Sciences, Faculty of Veterinary Medicine, University of Tehran, Tehran 1419963111, Iran
}

\begin{abstract}
Camel contagious ecthyma (CCE) has been reported in Mongolia, Kenya, Somalia and Sudan. CCE is caused by a Parapoxvirus affecting young animals by producing lesions around the lips and nostrils. The generalized form of CCE is uncommon. The aim of this study was to submit the first report of contagious ecthyma in Iran and also to help clinician to diagnose this disease with heavy economic losses easier. In the paper, an outbreak of CCE in dromedary and bactrian camels in Qom province of Iran was described and clinical signs were observed in one camel herd in October 2009. Nodules and scabs from seven affected animals were collected for virus identification. Total extracted DNA was used for polymerase chain reaction (PCR) to amplify a fragment of Parapoxvirus B2L gene. Results showed that camel calves $(n=27)$ less than one year old and one male bactrian camel were affected (no adult female camels were found to be infected). The prevalence of the disease in the herd, adult camels and camel calves was $30.33 \%, 1.5 \%$ and $100 \%$, respectively. Affected animals showed the swelling of head with nodular lesion around the lips. It then developed to pustules and fissured crusts. Previous involvement with this disease, history of contact with sheep or goats, food resources and season all can have a role in epidemiology of the disease.
\end{abstract}

Key words: Contagious ecthyma, first report, camel, Iran, PCR.

\section{Introduction}

There is only a little information on camel diseases compared to other species of animals. This may be mainly due to the fact that camel production is usually practiced on a migratory system in remote areas with harsh living conditions that make such studies difficult and expensive to execute [1].

Camels are susceptible to many infectious diseases, some of which have been investigated extensively because they also affect other species of farm animals. Such diseases include trypanosomiasis, anthrax, hemorrhagic septicemia, brucellosis, mange and pox [2]. Pox and pox-like diseases of camels are a group

Corresponding author: Mohammad Reza Mohebbi, Ph.D., research field: internal medicine. of exanthematous skin conditions, which recently emerge as being of increasing economic importance [3]. They may be caused by three distinct viruses: Orthopoxvirus cameli (camel pox), Parapoxvirus (camel contagious ecthyma (CCE)) and Papillomavirus (camel papilloma virus infection) [3]. The clinical signs of camel pox (caused by Orthopoxvirus cameli), CCE and camel papillomatosis are similar and can be confused, especially in the generalized form [4-6] and so far can be distinguished only by virus identification by electron microscopy or polymerase chain reaction (PCR). CCE was first described in Kazakhstan in 1968 [7]. Contagious ecthyma in camels (Camelus dromedarius) is a Parapoxvirus disease which has 
been described in many countries, such as Mongolia [8, 9], Somalia [4], the former Soviet Union [10], Libya [11], UAE [12], Saudi Arabia [13], Sudan [14] and Kenya [5].

The agent is a DNA poxvirus of the Parapoxvirus subgroup, which includes the closely related viruses' pseudocowpox (another cause of orf, like "milker's nodules" in humans), the agent of bovine papular stomatitis (BPS), Parapoxvirus of red deer in New Zealand, squirrel Parapoxvirus and Parapoxvirus of gray seals [15]. There are biological and genetic differences among strains of contagious ecthyma virus, so that cross protection may be limited and vaccines need to target the field strain. The virus is epitheliotropic, usually creating proliferative lesions in the skin of the lips, nostrils, oral mucosa, teats and occasionally the vulva [15].

The disease is characterized by localized lesions, although generalized forms have also been observed, resembling true camel pox [3]. Clinically, nodules appeared on the lips of affected animals, followed in most cases with swelling of the face and sometimes the neck [1]. Papules and vesicles appeared later and within a few days developed into thick scabs and fissured crusts [1]. Lesions occurred sometimes on the face, eyes and nares and in severe cases on the gingival, dental pad and tongue. Death was probably due to starvation caused by the inability of affected animals to graze or to suckle their dams. Healing occurred within 20-30 d in most cases, but sometimes the course of the disease extended up to three months [1]. Secondary bacterial infection or myiasis of affected parts may occur [15]. The possible involvement of insect transmission cannot be excluded. The disease seems to have a seasonal prevalence of an arthropod-borne disease. It appears in early rainy seasons and usually disappears by the end of raining [1]. Orf virus is transmissible to camels, but in comparison with sheep, camel orf infection has received little attention, apart from a limited number of reports describing its clinical and histological features.

It has been assumed that natural infections on pasture are the result of invasion of the virus after skin damage induced by prickly plants or stubble; application of a viral suspension to scarified skin is the established method of inducing orf [16]. Damage to the skin is essential for the establishment of orf infection and the development of typical lesions [16]. The thorny plants damaged the lips, allowing the transmission of Parapaxvirus [17]. Definitive diagnosis usually involves identifying the distinctive cross-hatched virus particles in typical early lesions with electron microscopy, PCR, immunohistochemistry or inoculation into known protected or susceptible animals [15]. Vaccination with the material containing CCE virus seemed to be promising. In contrast, camels were not protected after immunization with vaccinia virus and a vaccine against sheep and goat contagious ecthyma [8].

The aim of this study was to submit the first report of contagious ecthyma in Iran and alarm for its economic losses in camel rearing industry. Also in current study, all epidemiological aspect of ecthyma in camel was drown to help vet agent to diagnose this disease easier.

\section{Materials and Methods}

\subsection{Herd and Area}

The herd was comprised of 89 camels, including 88 dromedary camels (62 adult females and 26 calves, less than one year old) and one bactrian camel. The male bactrian camel was about three years old. It was bought purposely for breeding and it was why it was grouped with the herd.

The area is in the East of Qom province with about $200 \mathrm{~km}^{2}$ width, an arid area with hot and dry climate. The temperature in the area fluctuates during the year, and the day had an average temperature higher than $19^{\circ} \mathrm{C}$ and an annual rainfall was less than $125 \mathrm{~mm}$.

The dominant vegetation in the region was comprised of the tamarisk and haloxylon trees. Camels had freely grazed in the area. A total of four 
herds were included in this study, where three herds were all camels and a herd was a goat within it.

\subsection{Examination}

Affected animals were carefully examined for clinical signs. During examination, rectal temperature, vital signs, body condition score (BCS), rumination, ruminal movements, dehydration state and state of anemia were recorded, and peripheral lymph node, eyes, lesions in lips and muzzle were examined. Additional data concerning age and sex of animals were recorded.

\subsection{Sampling}

Biopsies from the lip lesions were collected in sterile containers for virological investigations. Samples were sent to Razi Vaccine and Serum Research Institute for diagnosis. Then, total extracted DNA was used for PCR to amplify a fragment of Parapoxvirus B2L gene [18]. The specific oligonucleotide primers used were designed according to Ref. [18].
The herd was monitored to investigate the epidemiological features of the disease.

\section{Results and Discussion}

\subsection{Results}

The calves were observed to have suffering from lesions on the lips and muzzle.

In these adult camels, lesions characterized by congested papules and scabs were seen on the lips and nostrils. The lesions were proliferative and were $3 \mathrm{~mm}$ to $2 \mathrm{~cm}$ in diameter. The animals were sensitive to palpation, thus often resulted in bleeding. In some cases, the mucosa of the mouth and nare were involved, showing haemorrhages in some of the affected parts. The lesions were limited in lips and muzzle, and other parts were not affected (Fig. 1). The animals got emaciated, because they lost their appetite, they were found to be slightly anemic and the ruminal movements as well as rumination decreased. As regards eye examination, conjunctivitis and epiphora were observed. Peripheral lymph nodes were mildly

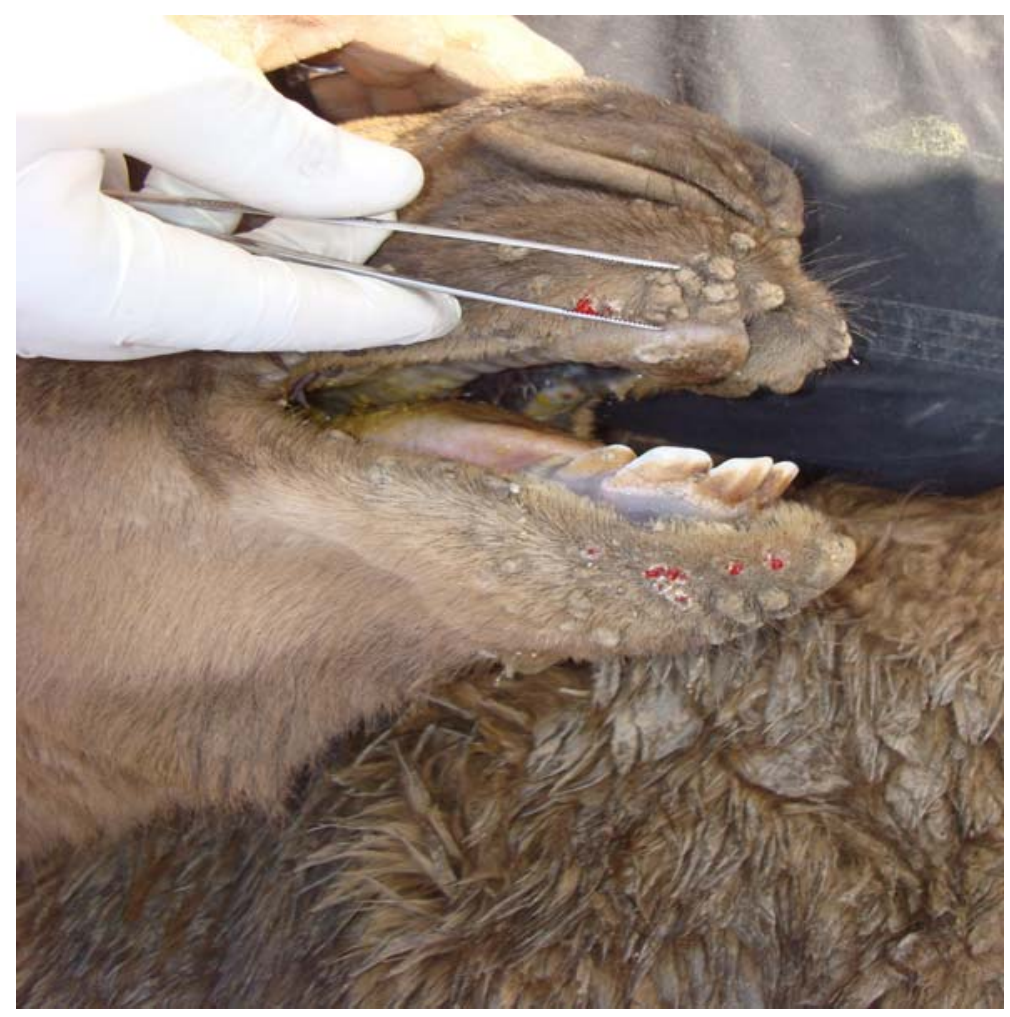

Fig. 1 Proliferative and hemorrhagic lesions with scabs in lips and muzzle in dromedary calves. 
enlarged. Affected animals had fever and average rectal temperatures reached $40.5{ }^{\circ} \mathrm{C}$. Furthermore, in some cases, the swelling of the head of the animal was observed. It could be noted that the bactrian camel was significantly affected compared to dromedary camel calves (Fig. 2). The lesions observed in the camels were also seen in the kids, although they received dose of pox vaccine, whereas the adult goat had no observable or clinical signs of lesions.

Congestion and hemorrhage in oral cavity were recorded in eight cases (29.6\%), and the swelling of the head was observed in five cases (18.5\%).

Diagnostic confirmation was done using PCR. Amplification of the B2L gene of camel skin biopsy samples showed a positive result of PCR product with the expected band size of 594 bp (Fig. 3).

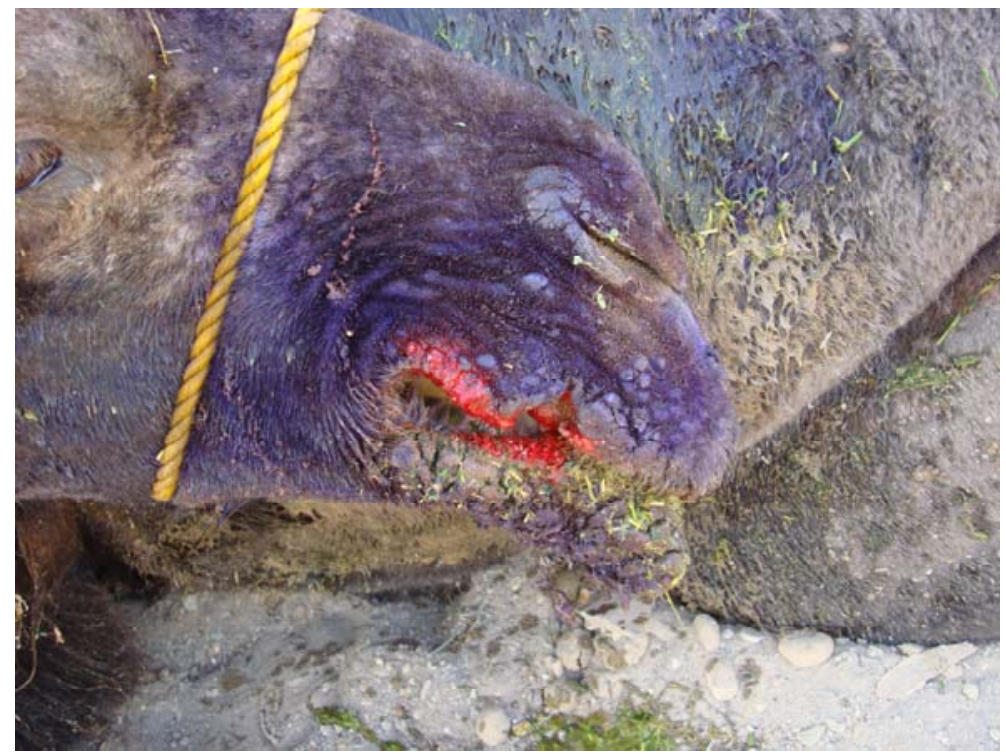

Fig. 2 Hemorrhagic popular lesions and fissured crusts in male adult bactrian camel.

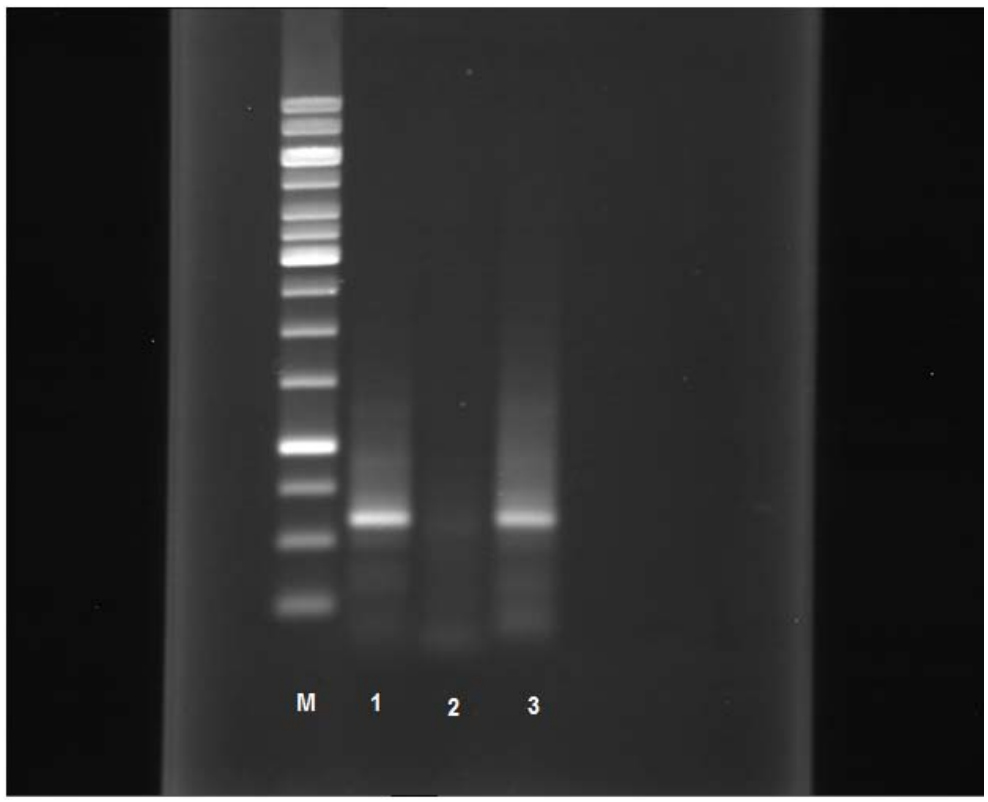

Fig. 3 Identification of CCE virus.

Lane M: 100 bp marker; lane 1: amplification of genomic LT cell DNA infected with contagious ecthyma virus, Kerman/2,000 strain; lane 2: amplification performed on normal skin biopsy; lane 3: amplification performed on skin biopsy with CCE infection. 
Table 1 Distribution of the disease based on age, breed, clinical signs and death.

\begin{tabular}{llll}
\hline Camels & No. & Numbers of having clinical signs & Numbers of death \\
\hline Calves (less than one year old) & 26 & 26 & 1 \\
Adult dromedary female & 62 & 0 & 0 \\
Adult bactrian male & 1 & 1 & 0 \\
\hline Total & 89 & 27 & 1 \\
\hline
\end{tabular}

The prevalence of the disease in the herd, adult camels and camel calves was $30.33 \%, 1.5 \%$, and $100 \%$, respectively (Table 1). The adult dromedary camels had no clinical signs of the diseases and all were healthy.

The mortality rates in whole herd, adults and calves were $1.12 \%, 0 \%$, and $3.84 \%$, respectively, and case fatality rates $(r)$ in these groups were $3.7 \%, 0 \%$, and $3.84 \%$, respectively.

The popular lesions progressed to proliferative lesions which were persisted for a week, and then the thick scabs appeared which were difficult to remove. They dried and fell down after 4-6 d, and totally healed in a month time. One animal died due to starvation and secondary infection.

Treatment with antibiotics was administrated only in bactrian camel due to secondary infection and respiratory disorder, which was given for $5 \mathrm{~d}$ and was also given a single dose of ketoprofen.

\subsection{Discussion}

Generally, the limited reports of orf infections in camels are difficult to compare due to the lack of good epidemiological tools as regards to study the infection rates.

The present study is the first report of CCE in Iran [19]. In an earlier study, clinical feature observed was the appearance of pustules on the nose, muzzle and lips and the enlargement of the lymph nodes [20]. Moallin et al. [21] recorded only localized lesions on the muzzle and lips. Similar signs have been reported in Somalia [21] and the Sudan [14]. In another study, generalized lesions involving the distal parts of the legs, the inner thighs and the vagina have also been reported [5]. In this study, also only localized proliferative lesions were founded and other parts were not affected.

In addition, same clinical stages were seen in whole herd, in different reports, it is probably related to the seasonal breeders and therefore many calves are born approximately at the same time.

Morbidity of $100 \%$ in calves had been described by other researchers, but this rate was reported to be low (10\%-20\%) in adult camels [5]. It was also reported that $98 \%$ of the cases of orf in camel occur in calves aged less than one year, with a mortality that reached $38 \%$ [22]. In another study [8], the morbidity in adults ranged from $10 \%$ to $80 \%$, in 2-month-old to 3-month-old suckling camels was between 50\%-70\% and it reached $100 \%$ in 1-year-old animals. The absence of contagious ecthyma in mature animals is probably due to an immune response, which is absent in very young camels; such a pattern has been described for goat kids [23].

In this study, only camel calves less than one year old and one adult bactrian male camel were affected. The adult dromedary camels remained unaffected, as they gained resistance being exposed to the affected animals. The bactrian male camel probably had not been exposed with CCE and therefore had not developed resistance to the disease and was not given vaccination against pox and pox-like viruses. The prevalence and mortality rate is similar to other studies.

Some reports have indicated that outbreaks of contagious ecthyma in camels have occurred in areas where orf is present in sheep [5, 24]. However, in another study in Saudi Arabia [25], camels were not observed to be cross-infected under field conditions, although they were in close proximity with sheep and goats. Gitao [20] reported that the common practice of keeping all camel calves in the same shelter at night 
could be responsible for the spread of the virus by contact, and he also proved that the outbreak in camel calves occurred when Parapoxvirus infections were also observed in goat kids who were raised in closed proximity place $[17,20]$. In the reported causes of the disease in camels, sheep and goats were proven to be carriers and non-carriers of contagious ecthyma. However, in this study, the herd together with the goat got infected.

In most studies, the annual young camel calf is exposed to infection when they start grazing prickly plants. This was the case in Asia [7] and Africa [14, 21, 22]. Factors responsible for this epizootiological feature seem to be the abrasion of the skin of the lips, resulting from that they ate thorny acacia trees at this time of the year when no other source of food was available [1]. The same opinion was also offered by Buchnev et al. [7], who argued that the thorny plants damaged the lips allowing transmission of Parapoxvirus.

In this study, tamarisk plant species could have been a contributory factor that transmitted the disease into the herd.

It is demonstrated that disease had a marked seasonality, being associated with the rainy season, and seemed to occur at this particular time every year $[1,7,21,26]$. This outbreak was observed in early winter (December) and season is also a big factor.

\section{Conclusions and Recommendations}

This study demonstrated that CCE has a considerable economic impact on camel production in Iran. The effect of this disease was not only due to mortality of camels but also the loss of weight and poor growth rate. Treatment of the infected animals is not easy, as it entails costly antibiotics and drugs. There is a need for an epidemiological study to confirm the presence of the disease. A comprehensive control and prevention program to minimize the occurrence of the disease is very necessary.

\section{References}

[1] Khalafalla, I. 1998. "Epizootiology of Camelpox, Camel Contagious Ecthyma and Camel Papillomatosis in the Sudan.” In Proceedings of the Third Annual Meeting for Animal Production under Arid Conditions, 115-31.

[2] McGrane, J. J., and Higgins, A. J. 1985. "Infectious Diseases of the Camel: Viruses, Bacteria and Fungi." $\mathrm{Br}$. Vet. J. 141 (5): 529-45.

[3] Munz, E. 1992. "Pox and Pox-Like Diseases of Camels." In Proceedings of the First International Camel Conference, 43-6.

[4] Jazek, Z., Kriz, B., and Rothbauer, V. 1983. "Camelpox and Its Risk to the Human Population.” J. Hyg. Epidemiol. Microbiol. Immunol. 27 (1): 29-42.

[5] Munz, E., Schillinger, D., Reimann, M., and Mahnel, H. 1986. "Electron Microscopical Diagnosis of Ecthyma Contagiosum in Camels (Camelus dromedarius): First Report of the Disease in Kenya.” J. Vet. Med. B 33 (1): 73-7.

[6] Munz, E., Moallin, A. S., Mahnel, H., and Reimann, M. 1990. "Camel Papillomatosis in Somalia." Zentralbl. Veterinarmed. B 37 (3): 191-6.

[7] Buchnev, K. N., Tulepbaev, S. Z., and Sansyzbaev, A. R. 1987. "Infectious Diseases of Camels in the USSR." Revue Sci. Tech. Off. Int. Epizoot. 6 (2): 487-95.

[8] Dashtseren, T. S., Solovyev, B. V., Varejka, F., and Khokhoo, A. 1984. "Camel Contagious Ecthyma (Pustular Dermatitis).” Acta Virol. 28 (2): 122-7.

[9] Khokhoo, A. 1982. "Biological Properties of Camel Contagious Ecthyma Virus.” M.Sc. thesis, Veterinary Institute, Brno.

[10] Roslyakov, A. A. 1972. "Comparison of the Ultrastructure of Camelpox Virus, the Virus of a Pox-Like Disease of Camels and Contagious Ecthyma Virus.” Vop. Virus. 17: 26-30. (in Russian)

[11] Azwai, S. M., Carter, S. D., and Woldehiwet, Z. 1995. "Immune Responses of the Camel (Camelus dromedurius) to Contagious Ecthyma (Orf) Virus Infection.” Veterinary Microbiology 47 (1-2): 119-31.

[12] Wernery, U., Kaaden, O. R., and Ali, M. 1997. “Orthopax Virus Infections in Dromedary Camels in United Arab Emirates (UAE) during Winter Season.” J. Camel. Prac. and Res. 4 (1): 51-5.

[13] Abu-Elzein, E. M. E., Coloyan, E. R., Gameel, A. A., Ramadan, R. O., and Al-Afaleq, A. I. 1998. "Camel Contagious Ecthyma in Saudi Arabia.” J. Camel Prac. and Res. 5: 225-8.

[14] Ali, O. A., Kheir, S. A., Abu-Damir, H., and Barri, M. E. 1991. "Camel (Camelus dromedarius) Contagious Ecthyma in the Sudan: A Case Report.” Rev. Elev. Méd. Vét. Pays Trop. 44 (2):143-5. 
[15] Bradford, P. S. 2015. "Diseases of the Alimentary Tract.” In Large Animal Internal Medicine, 5th ed.. Amsterdam: Elsevier, 748-50.

[16] Radostits, O. M., Gay, C. C., Hinchcliff, K. W., and Constable, P. D. 2007. "Diseases Associated with Viruses and Chlamydia.” In Veterinary Medicine: A Textbook of the Diseases of Cattle, Horses, Sheep, Pigs and Goats, 10th ed.. New York: Saunders Ltd., 1418-21.

[17] Wernery, U., and Kaaden, O. R. 2002. "Viral Infection Causing Diseases.” In Infection Diseases in Camelids, 2nd ed.. Berlin: Blackwell Science, 187-92.

[18] Inoshima, Y., Morooka, A., and Sentsui, H. 2000. "Detection and Diagnosis of Parapoxvirus by the Polymerase Chain Reaction.” J. Virol. Methods 84 (2): 201-8.

[19] Barani, S. M., Varshovi, H. R., Nikjou, D., and Niasari-Naslaji, A. 2011. "An Outbreak of Contagious Camel Ecthyma in Dromedary and Bactrian Camel in Qom, Iran.” In Proceedings of the 1st International Congress of Large Animal Practitioners, 173.

[20] Gitao, C. G. 1994. “Outbreaks of Contagious Ecthyma in
Camels (Camelus dromedarius) in the Turkana District of Kenya.” Rev. Sci. Tech. 13 (3): 939-45.

[21] Moallin, A. S. M., and Zessin, K. H. 1988. "Outbreak of Camel Contagious Ecthyma in Central Somalia.” Trop. Anim. Health Prod. 20 (3): 185-6.

[22] Khalafalla, A. I. 2000. "Camel Contagious Ecthyma: Risks in Young Calves.” Rev. Elev. Med. Pays Trop. 53 (2): 173-6.

[23] Robinson, A. J., and Balassu, T. C. 1981. "Contagious Pustular Dermatitis (Orf).” Vet. Bull. 51 (10): 771-80.

[24] Robertson, A. 1976. Handbook on Animal Diseases in the Tropics, 3rd ed.. London: British Veterinary Association, 9-11.

[25] Housawi, F., Abu-Elzein, E., Gameel, A., Mustafa, M., and Al-Afaleq, A. I. 2004. "Severe Auzdyk Infection in One-Month-Old Camel Calves (Camelus dromedarius).” Veterinarski Arhiv 74 (6): 467-74.

[26] Khalafalla, A. I., Agab, H., and Abbas, B. 1994. “An Outbreak of Contagious Ecthyma in Camels (Camelus dromedarius) in Eastern Sudan.” Trop. Anim. Health Prod. 26 (4): 253-4. 